\title{
Middle cerebral artery infarction in a cancer patient: a fatal case of Trousseau's syndrome
}

\author{
Peter YM Woo, Danny TM Chan, Tom CY Cheung, XL Zhu, WS Poon *
}

\section{A B S T R A C T}

Trousseau's syndrome is defined as any unexplained thrombotic event that precedes the diagnosis of an occult visceral malignancy or appears concomitantly with a tumour. This report describes a young, previously healthy man diagnosed to have an acute middle cerebral arterial ischaemic stroke and lowerlimb deep vein thrombosis, who subsequently succumbed to pulmonary arterial embolism. During the course of his illness, he was diagnosed to have a malignant pleural effusion secondary to an occult adenocarcinoma. This report highlights the need for a high degree of suspicion for occult malignancy and non-bacterial thrombotic endocarditis in young $(<60$ years old) ischaemic stroke patients with no identifiable conventional cardiovascular risks. In selected patients, transoesophageal echocardiography is the diagnostic investigation of choice, since transthoracic imaging is not sensitive. Screening tests for serum tumour markers and prompt heparinisation of these patients are suggested whenever ischaemic stroke secondary to malignancy-induced systemic hypercoagulability is suspected.

\section{Hong Kong Med J 2014;20:74-7}

DOI: $10.12809 / \mathrm{hkmj} 133780$

\author{
PYM Woo, MB, BS, MRCS (Edin) \\ ${ }^{1}$ DTM Chan, MB, ChB, FRCS (Edin) \\ ${ }^{2}$ TCY Cheung, MB, ChB, FRCR \\ ${ }^{1}$ XL Zhu, BMed (Jinan) FRCS (Edin) \\ ${ }^{1}$ WS Poon *, MB, ChB (Glasg), FRCS (Edin) \\ Division of Neurosurgery, Department of Surgery \\ 2 Department of Imaging and Interventional Radiology \\ Prince of Wales Hospital, The Chinese University of Hong Kong, Shatin, \\ New Territories, Hong Kong:
}

*Corresponding author: wpoon@cuhk.edu.hk

\section{Case report}

A 37-year-old Korean man, who was a non-smoker and previously healthy, experienced a sudden-onset right hemiparesis in April 2011. The patient was travelling in Guangzhou, China and was hospitalised within 4 hours of symptom onset. He was diagnosed to have a left middle cerebral artery (MCA) territory massive infarct based on plain computed tomography (CT). Contrast-enhanced magnetic resonance arteriography indicated a left proximal MCA occlusion (Fig a). The patient was treated conservatively and neither thrombolytic therapy nor operative management was given. Three days later, he was transferred to Hong Kong.

On examination the patient had expressive aphasia and right hemiplegia. He was able to obey commands, had a Glasgow Coma Scale score of 11/15 (E4V1M6) and was afebrile. His blood pressure was not high and his pulse was regular, nor were carotid bruits or heart murmurs detected.

Repeat CT of the brain showed a massive left MCA territory infarction with haemorrhagic conversion and a chest X-ray revealed a left pleural effusion (Figs 1b-d). An electrocardiogram (ECG) showed no arrhythmias and initial blood test results showed a raised white cell count of $15.4 \times 10^{9} / \mathrm{L}$ with neutrophilia. Other than that, the complete blood count, fasting serum glucose, lipid profile, and autoimmune markers were all within normal limits. The patient was initially managed medically with close neurological observations. In view of the neutrophilia, the patient was provisionally diagnosed to have bacterial endocarditis with complicating chest infection and parapneumonic pleural effusion. An echocardiogram was arranged and empirical intravenous antibiotics started.

Four days later, the patient became increasingly drowsy and an emergency decompressive craniectomy and duraplasty was performed (Figs 1e-f). The procedure was uneventful and the patient was stabilised.

As for delineating the cause for the stroke, all specimen culture results showed no evidence of an underlying infection. Percutaneous aspiration of the pleural effusion and pleural biopsy were performed. The biopsy showed no evidence of malignancy, but fluid cytology yielded adenocarcinoma cells. Serum tumour markers, including carcinoembryonic antigen and alpha-fetoprotein, were normal. The patient developed sinus tachycardia with an ECG showing a right heart strain pattern, before further cardiological tests (positron emission tomography and echocardiogram) could be performed. Blood for troponin-T and D-dimer levels were also elevated. The patient was subsequently diagnosed to have bilateral lower-limb deep vein thrombosis (DVT) extending from the external iliac vein to the popliteal vein. Thoracic CT was consistent with pulmonary embolism to the right lower lobe pulmonary arteries.

Subcutaneous low-molecular-weight heparin 
was started and an inferior vena cava (IVC) filter was placed. The patient's condition continued to deteriorate and he developed disseminated intravascular coagulopathy and respiratory failure. Progression of the pulmonary embolism was suspected, but the patient was deemed unsuitable for systemic thrombolysis due to his recent neurosurgery. The patient succumbed 2 weeks after the operation. A post-mortem examination was arranged, but waived by the coroner upon the family's request. The cause of death was pulmonary embolism contributed by an occult malignancy.

\section{Discussion}

Ischaemic stroke is the second most frequent neurological finding after brain metastases in postmortem studies of cancer patients. ${ }^{1}$ From an autopsy study, cerebral infarction was observed in $15 \%$ of 3426 patients diagnosed with cancer, of whom half were previously symptomatic. ${ }^{1}$ In a recent singlecentre retrospective review of 5106 patients admitted for ischaemic stroke, $24(0.47 \%)$ were diagnosed to have an underlying malignancy. In this subgroup

\section{一名癌症患者的大腦中動脈梗死：Trousseau 綜合徵的致命病例}

胡日明、陳達明、張智欣、朱獻倫、潘偉生

Trousseau綜合徵是指於診斷患有隱性惡性腫瘤前形成的血栓塞, 或於 確診腫瘤同時發現的血栓塞, 而非由其他病因引致。本文報告一名向 來健康良好的年輕患者, 被診斷為急性大腦中動脈缺血性中風及下肢 深靜脈血栓塞, 病榻過程中亦被診斷患有隱性腺癌並繼發惡性胸腔積 液, 最終因肺動脈栓塞致死。本文指出如年輕 ( 少於 60 歲) 的缺血性 中風患者無明確的心血管病, 便應高度警覺患有隱性惡性腫瘤和非細 菌性血栓性心內膜炎的可能。由於經胸壁心藏超聲波的影像敏感度不 足, 本報告中部份患者會使用經食道心臟超聲波作為診斷工具。如懷 疑患者因惡性腫瘤而導致血液高凝狀態並繼發缺血性中風, 建議可測 試血清腫瘤標誌物, 並馬上給與肝素注射療程。

of patients, the mean age was relatively young (52 years) and there was a lower incidence of the typical vascular risk factor profiles as noted in larger stroke cohort studies. $^{2}$

Trousseau first described the association
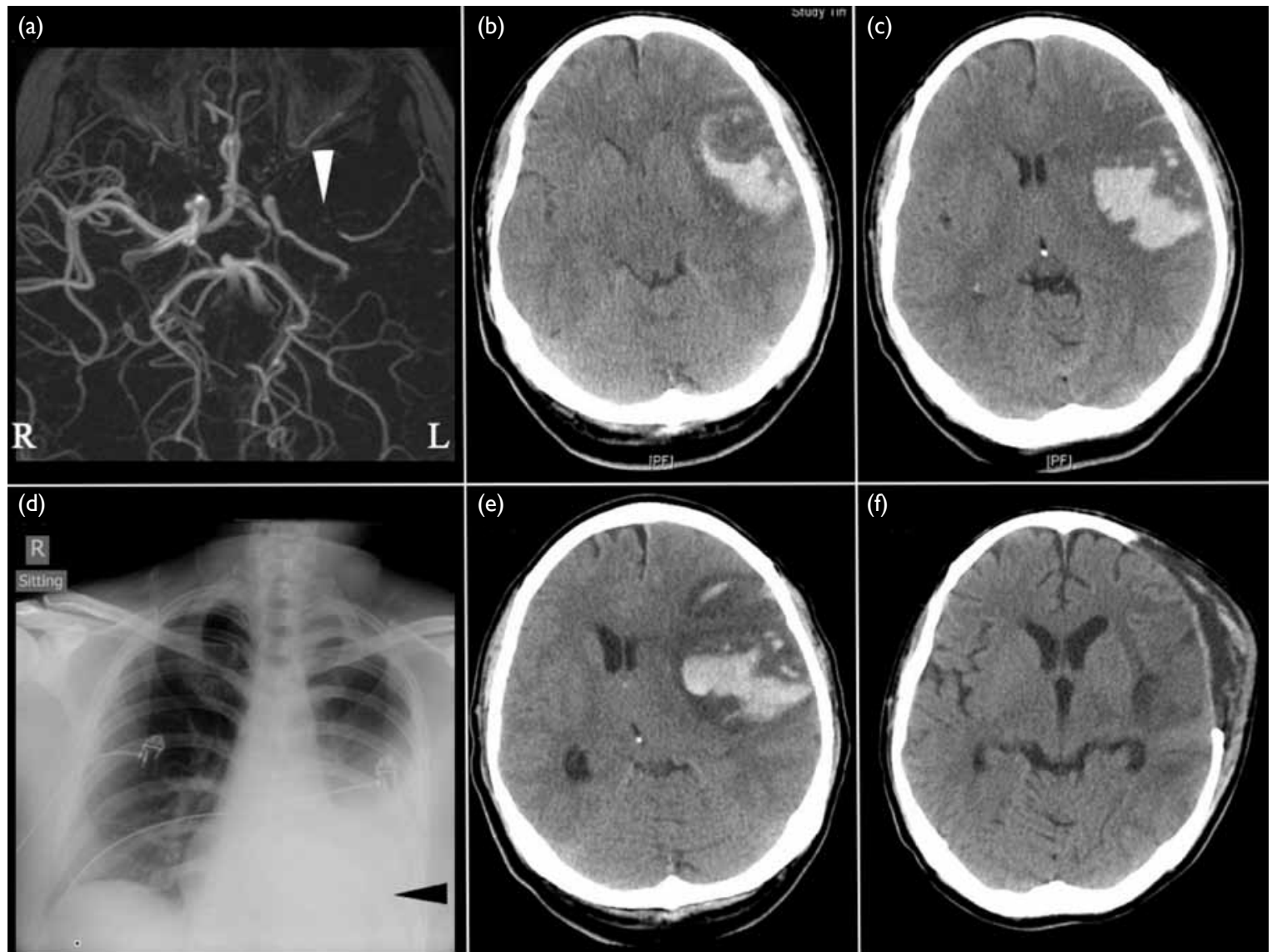

FIG. (a) Contrast-enhanced magnetic resonance angiograms on the day of symptom onset showing a left proximal middle cerebral artery (MCA) occlusion (white arrowhead). Brain computed tomography (CT) conducted 4 days later revealing haemorrhagic conversion of a left MCA region infarction with obliteration of (b) the basal cisterns and (c) midline shift. (d) Evidence of a left pleural effusion (black arrowhead) is present on the admission chest X-ray. (e) Deterioration in consciousness 8 days after symptom onset correlated with increased midline shift secondary to increased peri-haematomal oedema. (f) The 2-week postoperative CT showing satisfactory decompression and resolution of the intracerebral haematoma 
between vascular thrombosis and cancer in his monograph of a peculiar migratory thrombophlebitis in 1865 and diagnosed the syndrome on himself 2 years later when he succumbed to gastric adenocarcinoma. ${ }^{3,4}$ There are several definitions for Trousseau's syndrome including "the occurrence of thrombophlebitis migrans with visceral cancer" and "spontaneous recurrent venous thromboses and/or arterial emboli caused by non-bacterial thrombotic endocarditis (NBTE) in a patient with malignancy". ${ }^{4}$ But a prima facie definition should be any "unexplained thrombotic event that precede[s] the diagnosis of an occult visceral malignancy or appear[s] concomitantly with the tumor".4

It is estimated that $15 \%$ of cancer patients will suffer from a thromboembolic event during the course of their illness and up to $50 \%$ have evidence of venous thromboembolism on post-mortem examination. ${ }^{5}$ The underlying pathophysiological mechanisms can be broadly classified as either due to the malignancy itself or as an iatrogenic complication of oncological treatment such as radiotherapy-induced arteriopathy. In turn, tumourrelated ischaemic cerebrovascular events can be due to systemic hypercoagulability as part of a paraneoplastic syndrome, tumour emboli secondary to vessel infiltration, or contiguous compression of an artery. For this patient the thoracic CT revealed no evidence of infiltration of mediastinal great vessels or a cardiac lesion, so the possibility of arterial tumour emboli was unlikely. Brain magnetic resonance imaging also did not show brain metastasis. The fact that the patient developed extensive vascular thrombosis with bilateral lower-limb DVT and pulmonary embolism in spite of an IVC filter suggests that the cause of the stroke may have been due to malignancy-associated hypercoagulability.

The biological basis for systemic coagulopathy in Trousseau's syndrome has yet to be defined. It is thought to result from a complex interplay of tumour cell secretion of procoagulants and the host's blood vessels. When macrophages interact with malignant cells, there is a release of cytokines such as interleukin-1, interleukin-6, and tumour necrosis factor that can lead to endothelial damage and thrombogenesis on viable surfaces. ${ }^{6}$ Tumour cells may also secrete tissue factor and cysteine proteases with thromboplastin-like properties that activate coagulation factors VII and X. Finally mucin-producing adenocarcinomas can lead to direct non-enzymatic activation of factor $\mathrm{X} .{ }^{7}$ In this state of hypercoagulability, the two commonest underlying mechanisms for ischaemic stroke are related to cardiogenic embolism from NBTE or cerebral intravascular coagulation. ${ }^{8}$

\section{Non-bacterial thrombotic endocarditis}

Up to half of patients with NBTE develop systemic emboli, most often to the cerebral vasculature and result in stroke, but some emboli also end up in the pulmonary, cardiac, and renal circulations. ${ }^{6}$ This may have been the underlying cause for the patient's massive MCA infarction and pulmonary embolism. Formally known as marantic endocarditis, the condition is characterised by sterile platelet and fibrin rich thrombi that form on previously undamaged heart valves. ${ }^{9}$ The incidence is largely unknown but according to Graus et al's autopsy series, ${ }^{1}$ it was noted in $1.6 \%$ of adult cancer patients. Often, it is encountered in patients with advanced-stage malignancies, in particular adenocarcinomas of the lung or gastro-intestinal tract; as in this patient, rarely NBTE can be a harbinger of occult cancer. ${ }^{8,10}$

Its pathogenesis is incompletely understood, but is possibly due to cytokine-mediated inflammatory valvular tissue injury that predisposes to thrombus formation. ${ }^{6}$ For several reasons, NBTE is difficult to diagnose. Thus, though aortic and mitral valves are the most commonly affected, patients seldom have detectable cardiac murmurs. ${ }^{6,11}$ Concomitantly, immunocompromised patients can suffer from infective endocarditis that confounds the diagnosis. Finally, transthoracic echocardiography is not sufficiently sensitive in detecting the smaller friable valvular vegetations of NBTE. ${ }^{12}$ Careful selection of patients for transoesophageal echocardiography, the preferred diagnostic test, is claimed to be preferable. ${ }^{6}$ Patients without end-stage malignancy, acceptable performance status of 3 or less on the Eastern Cooperative Oncology Group scale and with nondebilitating stroke are suitable candidates. For this patient, the diagnosis of NBTE was inferred in view of the negative culture and serology results and failed response to systemic antibiotics.

Treatment is directed at the underlying malignancy coupled with systemic anticoagulation. Since patients often present with metastatic disease, curative options are limited and anticoagulation should be administered indefinitely, since thromboembolic events tend to recur after discontinuation. ${ }^{6}$ Unfractionated heparin, given either intravenously or subcutaneously, has been proven to be most effective. ${ }^{6}$ Alternatively, lowmolecular-weight heparin could be considered, but evidence about its utility is anecdotal. In contrast, vitamin $\mathrm{K}$ antagonists such as warfarin are not recommended, as recurrent thromboembolic events are common and expose the patient to unnecessary bleeding risks. ${ }^{6,13}$ The exact reason for warfarin resistance is unknown, but it has been suggested that thrombosis secondary to cytokine-induced inflammation is preferentially mediated by nonvitamin-K-dependent coagulation factors. ${ }^{13}$

\section{Cerebral intravascular coagulation}

Cerebral intravascular coagulation is a post- 
mortem pathological diagnosis made when cardiac valvulopathy is excluded. Originally described in autopsy studies among patients with leukaemia and breast cancer, this condition involves diffuse fibrinous occlusions of small cerebral blood vessels leading to micro-infarcts. Patients often present with progressive encephalopathy and seldom develop focal neurological deficits, although partial seizures have been reported. ${ }^{14}$ No reliable laboratory or radiological investigations are available to detect this disorder. Patients are usually in their preterminal state and management is often supportive. ${ }^{8}$

\section{Conclusion}

This patient suffered from a fatal manifestation of Trousseau's syndrome. One should be cognisant of the diagnosis of NBTE as a cause of ischaemic stroke in any patient with a known or suspected underlying malignancy. This is particularly true if new heart murmurs are detected in a cancer patient. Conversely, for a young patient $(<60$ years old), ischaemic stroke with no overt vascular risk factors should be considered for cancer screening. In selected cases, transoesophageal echocardiography appears to be the investigation of choice for NBTE. Anticoagulation, preferably with unfractionated heparin, should be administered as soon as possible in ischaemic stroke patients whenever such an event is a suspected consequence of an occult or overt malignancy causing systemic hypercoagulability.

\section{References}

1. Graus F, Rogers LR, Posner JB. Cerebrovascular complications in patients with cancer. Medicine (Baltimore) 1985;64:16-35.
2. Taccone FS, Jeangette SM, Blecic SA. First-ever stroke as initial presentation of systemic cancer. J Stroke Cerebrovasc Dis 2008;17:169-74.

3. Trousseau A. Lectures on clinical medicine, delivered at the Hotel-Dieu, Paris. 2nd ed. Philadelphia: Lindsay \& Blakiston; 1865: 281-332.

4. Varki A. Trousseau's syndrome: multiple definitions and multiple mechanisms. Blood 2007;110:1723-9.

5. Deitcher SR. Cancer and thrombosis: mechanisms and treatment. J Thromb Thrombolysis 2003;16:21-31.

6. el-Shami K, Griffiths E, Streiff M. Nonbacterial thrombotic endocarditis in cancer patients: pathogenesis, diagnosis, and treatment. Oncologist 2007;12:518-23.

7. Bick RL. Cancer-associated thrombosis. N Engl J Med 2003;349:109-11.

8. Rogers LR. Cerebrovascular complications in cancer patients. Neurol Clin 2003;21:167-92.

9. Gross L, Friedberg CK. Nonbacterial thrombotic endocarditis. Classification and general description. Arch Intern Med (Chic) 1936;58:620-40.

10. Edoute Y, Haim N, Rinkevich D, Brenner B, Reisner SA. Cardiac valvular vegetations in cancer patients: a prospective echocardiographic study of 200 patients. Am J Med 1997;102:252-8.

11. Rosen P, Armstrong D. Nonbacterial thrombotic endocarditis in patients with malignant neoplastic diseases. Am J Med 1973;54:23-9.

12. Lee RJ, Bartzokis T, Yeoh TK, Grogin HR, Choi D, Schnittger I. Enhanced detection of intracardiac sources of cerebral emboli by transesophageal echocardiography. Stroke 1991;22:734-9.

13. Bell WR, Starksen NF, Tong S, Porterfield JK. Trousseau's syndrome. Devastating coagulopathy in the absence of heparin. Am J Med 1985;79:423-30.

14. Collins RC, Al-Mondhiry H, Chernik NL, Posner JB. Neurologic manifestations of intravascular coagulation in patients with cancer. A clinicopathologic analysis of 12 cases. Neurology 1975;25:795-806. 